\section{Oncoplastic surgery in elderly patients with breast cancer: overtreatment or a goal worth pursuing?}

\author{
Stefano Chiodi, ${ }^{1}$ Ugo Catrambone, ${ }^{2}$ \\ Giuseppe Verrina, ${ }^{1}$ Andrea Decensi, ${ }^{3}$ \\ Pamela Guglielmini, ${ }^{4}$ Marco Filauro ${ }^{2}$ \\ 'Department of Maxillofacial and \\ Plastic-Reconstructive Surgery, Galliera \\ Hospital, Genova; ${ }^{2}$ Department of \\ General and Epatobiliopancreatic \\ Surgery, Galliera Hospital, Genova; \\ ${ }^{3}$ Department of Medical Oncology, \\ Galliera Hospital, Genova; ${ }^{4}$ Department \\ of Medical Oncology, SS. Antonio e \\ Biagio e C. Arrigo Hospital, Alessandria, \\ Italy
}

\section{Abstract}

Breast cancer is the most common cancer in women in Western countries, which increases with age. The improvement of reconstructive methods in light of the principles and techniques borrowed directly from cosmetic surgery has helped raise the quality in terms of aesthetic results in conservative treatment. This approach has reached results, which merits a more precise role of self-autonomy and the name of oncoplastic breast surgery. Today this approach is becoming, in the centers dedicated to the treatment of breast cancer, the gold standard in the surgical treatment of patients with this cancer. So if the role of oncoplastic in the surgical treatment of breast cancer is to be established, it remains crucial to have a selection of patients who could benefit from this approach: today, age is one of the determining factors in the selection of patients and, in fact, patients over 75 years, are often excluded from surgery of this type. In our opinion, after a multidisciplinary assessment, also the older women could be able to receive this type of surgical approach.

\section{Introduction}

Breast cancer is the most common cancer in women in Western countries, which increases with age. The need for surgical demolition, (derived from the paradigm of the maximum tolerable treatment), has led to some patients who are suffering from this disease to be disfigured. This often causes the patients to alter their lives specifically from the point of view of work, psychological prob- lems and relationships. Add to this the fact that the fear of mastectomy has, for years, made patients refuse early detection mechanisms and even caused women to deny the existence of a tumor results in a much more difficult aspect to treatment.

In recent years there has been increasing attention about this issue, which has created a new Surgical philosophy in regards to the treatment of breast cancer. The n ew paradigm of minimum effective treatment is now being sought. There has been a gradual development of less invasive interventions with a decrease in the total number of mastectomies carried out. Surgeries such as mastectomy with limited skin savings and nipple areola complex, quadrantectomy associated with radiotherapy, tumorectomy enlarged lymph node biopsies and targeted have all decreased. ${ }^{1-4}$ The improvement of reconstructive methods in light of the principles and techniques borrowed directly from cosmetic surgery, has helped raise the quality in terms of aesthetic results in conservative treatment. ${ }^{5,6}$ This close association between radical surgery and reconstructive breast surgery has reached levels and results which merits a more precise role of self autonomy and the name of oncoplastic breast surgery. Today this approach is becoming, in the centers dedicated to the treatment of breast cancer, the gold standard in the surgical treatment of patients with this cancer.

This includes a multi-specialist team, which not only involves the surgeon general and the plastic surgeon in particular but also the medical oncologist and the radiation oncologist. Collaboration starts from the time of diagnosis with the evaluation of anatomical and clinical aspects, such as the size and characteristics of the tumor, the age and general condition of the patient. These elements also include the emotional aspects related to the diagnosis of the disease and the expectations of the individual patient. After all considerations an operative program which works best for each woman should be organized with the principal aim of curing the cancer. However in reference to the first paragraph it should also aim to restore an overall aesthetic harmony of the breast region.

So if the role of oncoplastic in the surgical treatment of breast cancer is to be established, it remains crucial to have a selection of patients who could benefit from this approach.

Also today, age is one of the determining factors in the selection of patients and, in fact, patients over 75 years, are often excluded from surgery of this type.

We present the case of a 77-year-old patient suffering from a locally advanced breast cancer who has undergone this type of surgical approach.
Correspondence: Stefano Chiodi MD, Department of Maxillofacial and Plastic-Reconstructive Surgery, Galliera Hospital, via delle Cappuccine 14,16128 Genova, Italy.

Tel.: +39.010 .5634238 - Fax: +39.010 .57481442$

E-mail: stefano.chiodi@galliera.it

Key words: Breast cancer in elderly; oncoplastic breast surgery; multidisciplinary assessment.

Contributions: the authors contributed equally.

Conflict of interests: the authors declare no potential conflict of interests.

Received for publication: 25 January 2016. Accepted for publication: 17 February 2016.

This work is licensed under a Creative Commons Attribution-NonCommercial 4.0 International License (CC BY-NC 4.0).

(C) Copyright S. Chiodi et al., 2016

Licensee PAGEPress, Italy

Geriatric Care 2016; 2:5776

doi:10.4081/gc.2016.5776

\section{Case Report}

We present the case of a 77-year-old female at time of diagnosis in good general condition, without co-pathology major, apart from high blood pressure medication and a body mass index $>27$.

In January 2015, the patient, after discovering a swelling in her right breast underwent a mammogram and breast ultrasound, which showed a lesion in the QIE of the right breast of about $1.3 \mathrm{~cm}$ infiltrating the dermis (Figures 1 and 2). A total body computed tomography was negative for disseminated disease. After a multidisciplinary assessment between the oncologist, surgeon and plastic surgeon she underwent surgery having a quadrantectomy inferiorright with sentinel node biopsy and mastoplasty in March 2015 (Figure 3). The histological examination showed macroscopically presence in two nodules one of $2.4 \mathrm{~cm}$ and the other $1 \mathrm{~cm}$ both microscopically results showed two moderately differentiated infiltrating ductal endocrine-responsive carcinomas (Luminal B). One of the two removed sentinel nodes tested positive for cancer with endo-lymphatic emboli endovascular - pathologic stage T2 p mp N1.

In June 2015 after a positive axillary lymph node test the patient was submitted for an ipsilateral axillary dissection (free from lymph nodes metastases) and, concurrently, the patient then underwent controlateral with unilateral breast reduction (Figure 4).

Postoperatively a positron-emission tomography test was preformed which proved negative for the presence of any active disease. 
Subsequently breast radiotherapy and adjuvant hormonal therapy was followed with positive results.

\section{Discussion and Conclusions}

The psychological and emotional benefits for the patient from having a more conservative surgical approach instead of a full mastectomy is now widely proven. However, data shows that $20-30 \%$ of patients who have had constructive breast surgery are not satisfied with the result. To remedy this problem, surgery Oncoplastic, able to combine plastic surgery techniques with those of surgical oncology, it is the gold standard of care in the majority of centers specialized for the treatment of breast cancer.

The incidence of breast cancer increases with age: about half of new cases occur in women over the age of 65 years and a third over 70 years. Furthermore it is estimated that in most Western countries the number of women over 65 years is likely to increase by about $50 \%$ over the next twenty years. Elderly patients suffering from this cancer tend to have worse survival statistics than younger patients. This increase in mortality is partly due to a delay in diagnosis, linked to a certain reticence on the part of women.

Older ladies appear to be more resistant to undergo screening tests, but also there seems to be a link to a widespread tendency to under treat elderly patients. This trend is not confined to more aggressive therapies such as chemotherapy, but emerges at the moment the decision about what is the best surgical approach should be for the patient occurs.

Today we know that chronological age is not an absolute restraint to a type of treatment, therapeutic choice will weigh on the biological age, life expectancy and co-diseases of the patient, elements that, together are able to contraindicate or influence the choice of treatment. Conservative surgical treatment is independent of age but is in relation to the stage of the disease and the relationship between the volume of the breast and that of the tumor as is the case for younger patients.

The rationale is simple, mastectomy in patients with advanced age and in poor condition and in these cases the choice is only cytoreductive in order to start the patient on complementary treatments. Mastectomy guarantees undoubtedly an excellent control locally of the disease, but do not forget that data from non-randomized studies, have shown a lower incidence of local recurrence after breast-conserving surgery and radiation therapy in elderly patients compared to younger.

As we suggested in our experience, many older patients prefer to keep the breast if the opportunity is offered however this is not always the case. With some physicians disregarding how this surgery will affect the bodies of these women. Their treatment is often demolition of the breast. In line with these considerations, the national and international guidelines recommend that treatments proposed for this age group are the same as those used in younger women, ${ }^{6,7}$ except for contraindications related to co-diseases or preference of the patient.

In a recent paper published this year in the British Journal of Surgery we were presented with data for a review of the literature on the use of techniques and reconstructive oncoplastic surgery in older women. ${ }^{8}$ The research included a total of nine studies, most of which were retrospective, (the study used smaller numbers and different parameters when they defined the word older patients).

From the data analysis it became clear that the outcome of patients undergoing oncoplastic surgery was equivalent to that of younger women, but showed a tendency not to propose this type of treatment to patients over 70 years for reasons related to an extension of the oper-

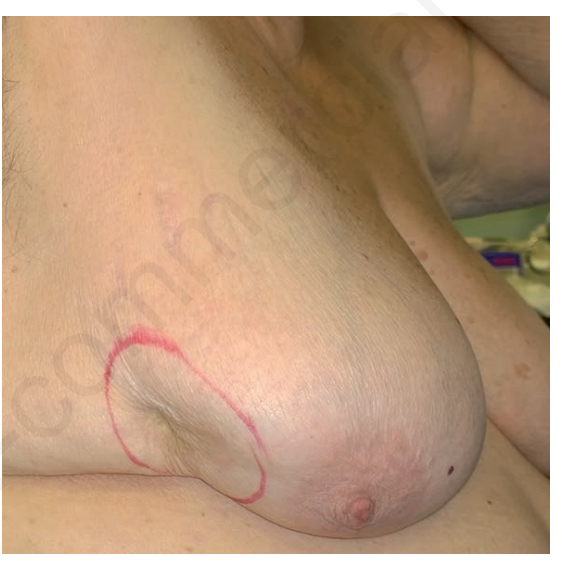

Figure 1. Ductal carcinoma in the lateral quadrants of the right breast infiltrating the dermis (lateral view).

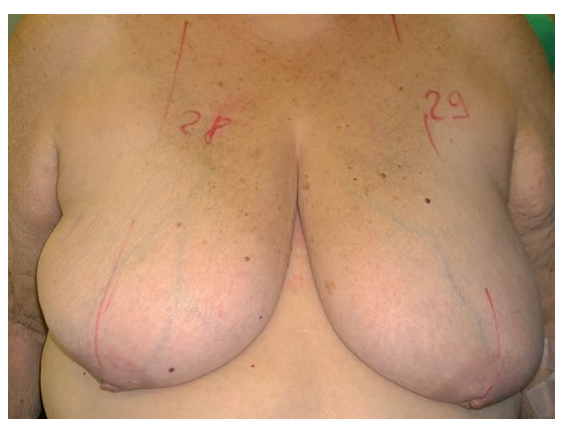

Figure 2. Ductal carcinoma in the lateral quadrants of the right breast infiltrating the dermis (frontal view). ative time, the lack of standard care etc., but especially the bias of surgeons about the perception of body image and their expectations by the patients. Only one of the studies directly mentioned oncoplastic techniques in the preservation of the breast. ${ }^{9}$ In this paper the authors concluded that Oncoplastic techniques were absolutely safe in patients in their seventies and even suggested a possible advantage in terms of aesthetic results using this approach in these women. This is due to higher fat content in the breast in the elderly women.

In the evaluation of safety it revealed no significant differences compared to younger patients in terms of post-surgical complications and age in itself did not prove a negative prognostic factor of survival. The studies demonstrated, that women who underwent this type of surgery have over the years experienced a better quality of life compared to those submitted for mastectomy.

A UK Parliamentary Commission report, which was published in 2013, dealt with the fact that the English have been under treating elderly patients suffering from breast cancer.

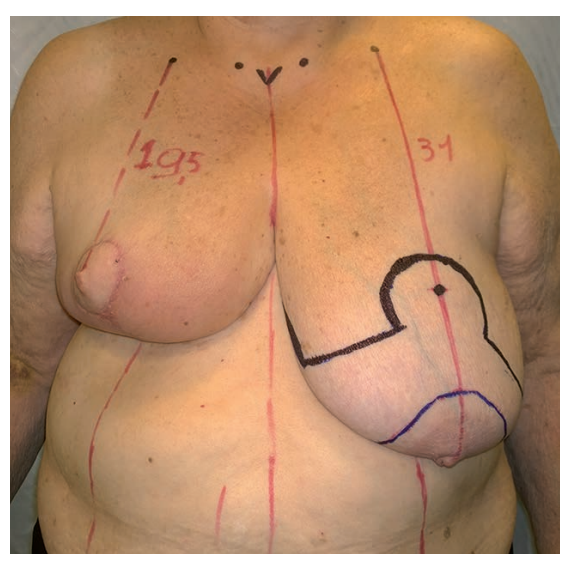

Figure 3. Inferior-right quadrantectomy with sentinel node biopsy and mammoplasty.

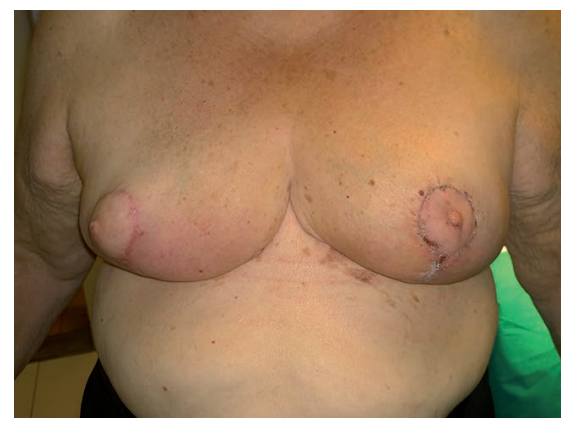

Figure 4. Controlateral inferior pedicle breast reduction: final result. 
This report was called age is only a number which was to emphasize that these women should receive the most appropriate treatment on the basis of general conditions and co-diseases and not only, as it happens in most cases, according to their age. ${ }^{10}$ Surely in patients with breast cancer over 75 it is crucial, perhaps even more than in the young, a multimodal treatment approach that is cut to measure and where the therapeutic strategy must be the result of proper multidisciplinary assessment.

\section{References}

1. Jakesz R, Samonigg H, Gnant M, et al. Significant increase in breast conservation in 16 years of trials conducted by the Austrian Breast and Colorectal
Study Group. Ann Surg 2003;237:556-64.

2. Fisher B, Dignam J, Wolmark $\mathrm{N}$, et al. Lumpectomy and radiation therapy for the treatment of intraductal breast cancer: findings from National Surgical Adjuvant Breast and Bowel Project B-17. J Clin Oncol 1998;16:441-52.

3. Veronesi U, Cascinelli N, Mariani L, et al. Twenty-year follow-up of a randomized study comparing breast-conserving surgery with radical mastectomy for early breast cancer. N Engl J Med 2002;347:1227-32.

4. Hamdi M, Wolfli J, Van Landuyt K. Partial mastectomy reconstruction. Clin Plast Surg 2007;34:51-62.

5. Losken A, Hamdi M. Partial breast reconstruction: current perspectives. Plast Reconstr Surg 2009;124:722-36.

6. Cawthorne S. All party parliamentary group on breast cancer: inquiry into older age and breast cancer. ABS Newsletter 2013:1.
7. Biganzoli L, Wildiers H, Oakman C, et al. Management of elderly patients with breast cancer: updated recommendations of the International Society of Geriatric Oncology (SIOG) and European Society of Breast Cancer Specialists (EUSOMA). Lancet Oncol 2012;13:e148-60.

8. James B, McCulley SJ, Macmillan RD. Oncoplastic and recontructive surgery in the ealderly. Br J Surg 2015;102:480-8.

9. De Lorenzi F, Rietjens M, Soresina M, et al. Immediate breast reconstruction in the elderly: can it be considered an integral step of breast cancer treatment? The experience of the European Institute of Oncology, Milan. J Plastic Reconstr Aesthetic Surg 2010;63:511-5.

10. All Party Parliamentary Group on Breast Cancer (APPGBC). Age is just a number. The Report of the Parliamentary Inquiry into Older Age and Breast Cancer. APPGBC: London; 2013. 